\title{
HOMOGENIZATION OF FIBER REINFORCED BRITTLE MATERIALS: THE EXTREMAL CASES
}

\author{
MARCO BARCHIESI \& GIANNI DAL MASO
}

\begin{abstract}
We analyze the behavior of a fragile material reinforced by a reticulated elastic unbreakable structure in the case of antiplane shear. The microscopic geometry of this material is described by means of two small parameters: the period $\varepsilon$ of the grid and the ratio $\delta$ between the thickness of the fibers and the period $\varepsilon$. We show that the asymptotic behavior as $\varepsilon \rightarrow 0^{+}$and $\delta \rightarrow 0^{+}$depends dramatically on the relative size of these parameters. Indeed, in the two cases considered, i.e., $\varepsilon \ll \delta$ and $\varepsilon \gg \delta$, we obtain two different limit models: a perfectly elastic model and an elastic model with macroscopic cracks, respectively.
\end{abstract}

Keywords: homogenization, free discontinuity, $\Gamma$-convergence, multiscale analysis, fracture mechanics.

2000 Mathematics Subject Classification: 35B27, 73B27, 73M25

\section{Contents}

1. Introduction

2. Problem setting

3. A lower bound estimate

4. The perfectly elastic case

5. The brittle case

\section{INTRODUCTION}

The aim of this paper is to study the asymptotic behavior of a fragile material reinforced by a reticulated elastic structure (the dark and the white part in Figure 1, respectively) in the case of antiplane shear. The reinforcement consists of thin unbreakable fibers disposed periodically along two orthogonal directions of the plane. Two parameters are involved: the period $\varepsilon$ of the grid and the ratio $\delta$ between the thickness of the fibers and the period $\varepsilon$.

We show that the overall behavior of the structure depends dramatically on these relative size of the parameters. If $\delta / \varepsilon \rightarrow+\infty$, the asymptotic behavior is perfectly elastic without cracks. Instead, if $\delta / \varepsilon \rightarrow 0^{+}$, we obtain in the limit an elastic material with macroscopic brittle cracks.

Let $\Omega \subseteq \mathbb{R}^{2}$ be the reference configuration, representing a cross section of the material. Since we are taking into account the presence of cracks, the natural mathematical setting for our problem is the space $G S B V(\Omega)$, introduced by De Giorgi and Ambrosio [9], for

Date: October 22, 2009. 


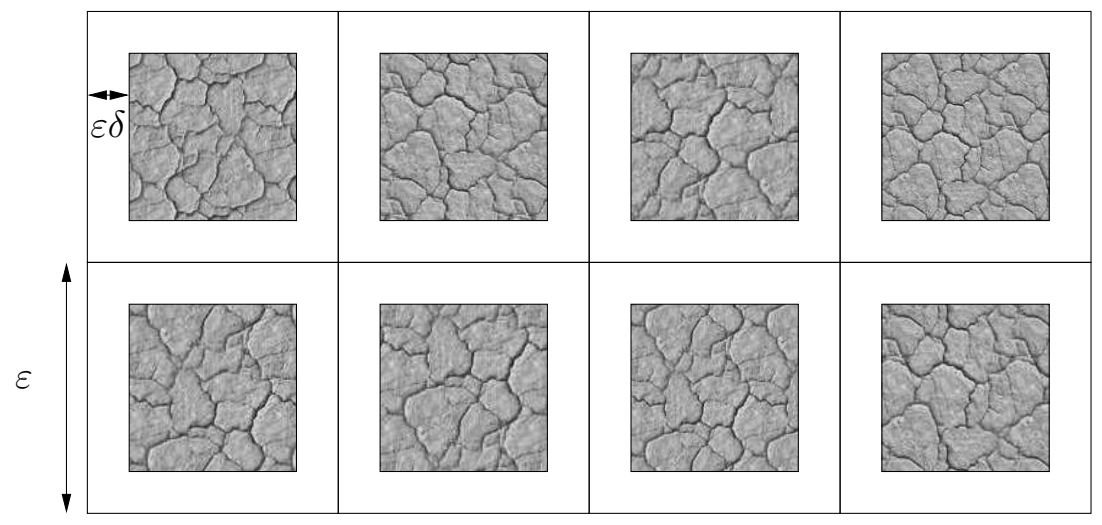

FiguRE 1. A representation of the composite material.

which we refer to Section 2. The behavior of the composite can be described by the following family of functionals:

$$
F_{\varepsilon}(u):= \begin{cases}\int_{\Omega}|\nabla u(x)|^{2} d x+\mathcal{H}^{1}\left(S_{u}\right) & \text { if } \quad S_{u} \subseteq \Omega_{\varepsilon} \\ +\infty & \text { otherwise }\end{cases}
$$

where $\Omega_{\varepsilon}:=\Omega \cap \varepsilon\left([\delta, 1-\delta]^{2}+\mathbb{Z}^{2}\right), \mathcal{H}^{1}$ is the one-dimensional Hausdorff measure, and $\delta=\delta(\varepsilon) \in(0,1 / 2)$.

The scalar function $u$ denotes the displacement, while its discontinuity set $S_{u}$ represents the crack. In addition to the integral over $\Omega$, which gives the standard stored energy for linear elasticity in the antiplane case, $F_{\varepsilon}$ includes a length term, which accounts for the energy needed to open the crack. The set $\Omega_{\varepsilon}$ represents the brittle region where the crack could develop, while the set $\Omega \backslash \Omega_{\varepsilon}$ represents the unbreakable fibers inside the material.

Our purpose is to determine the $\Gamma$-limit $F_{\text {hom }}$ of $F_{\varepsilon}$ as $\varepsilon \rightarrow 0^{+}$and to distinguish the different asymptotic models according to the limit $\vartheta$ of $\delta(\varepsilon) / \varepsilon$. More precisely, we analyze the extremal cases

$$
\vartheta=+\infty \quad \text { and } \quad \vartheta=0 .
$$

In the first case, we show that

$$
F_{\text {hom }}(u)= \begin{cases}\int_{\Omega}|\nabla u(x)|^{2} d x & \text { if } u \in H^{1}(\Omega), \\ +\infty & \text { otherwise. }\end{cases}
$$

This functional describes a material without cracks. Indeed, even if there are microscopic cracks in the material at scale $\varepsilon$, they cannot merge into a macroscopic crack and have no effect on the limit, since the elastic fibers separate the brittle regions in an effective way.

In the second case we prove that

$$
F_{\text {hom }}(u)=\int_{\Omega}|\nabla u(x)|^{2} d x+\mathcal{H}^{1}\left(S_{u}\right) .
$$

This means that, despite the presence of the unbreakable fibers, the collective behavior of microscopic cracks is equivalent in the limit to a macroscopic crack. 
In the intermediate case $\vartheta \in(0,+\infty)$, under the assumption that the $\Gamma$-limit has an integral representation, we obtain that the surface term depends also on the size $[u]$ of the jump.

Remark 1.1. Homogenization in $S B V$ setting has been developed in previous works [5, 3]. Yet, these results do not apply to our case, because the surface energy in (1.1) does not satisfy their hypotheses.

Remark 1.2. Another interesting situation occurs when the parameter $\delta$ is fixed and independent of $\varepsilon$ and the family of functionals has the form

$$
F_{\varepsilon}(u):= \begin{cases}\int_{\Omega}|\nabla u(x)|^{2} d x+a(\varepsilon) \mathcal{H}^{1}\left(S_{u}\right) & \text { if } \quad S_{u} \subseteq \Omega_{\varepsilon}, \\ +\infty & \text { otherwise, }\end{cases}
$$

where $a(\varepsilon) \rightarrow 0^{+}$as $\varepsilon \rightarrow 0^{+}$. In this case the asymptotic behavior as $\varepsilon \rightarrow 0^{+}$leads to a damage problem, recently studied in $[12,13]$.

\section{Problem setting}

In the following we assume that $\Omega$ is a bounded open subset of $\mathbb{R}^{2}$ with Lipschitz boundary. We recall here some notation about the space of special functions of bounded variation on $\Omega$, briefly $S B V(\Omega)$, and we refer to [1] for the definitions and the standard theory.

If $u \in S B V(\Omega)$,

- $D u$ is the distributional gradient of $u$,

- $\nabla u$ is the approximate gradient of $u$,

- $S_{u}$ is the approximate discontinuity set of $u$,

- $\nu_{u}$ is the generalized normal to $S_{u}$,

- $u^{ \pm}$are the traces of $u$ on both sides of $S_{u}$,

- $[u]:=\left|u^{+}-u^{-}\right|$is the size of the jump.

The same notation is used when $u \in G S B V(\Omega)$, the space of generalized special functions of bounded variation on $\Omega$. We recall that $u \in G S B V(\Omega)$ if and only if $u: \Omega \rightarrow \mathbb{R}$ is measurable and $(u \wedge m) \vee(-m) \in S B V(U)$ for every $m \in \mathbb{N}$ and every open set $U$ with $\bar{U} \subseteq \Omega$.

We shall frequently use the spaces

$$
\begin{aligned}
& S B V^{2}(\Omega):=\left\{u \in S B V(\Omega): \nabla u \in L^{2}\left(\Omega, \mathbb{R}^{2}\right) \text { and } \mathcal{H}^{1}\left(S_{u}\right)<+\infty\right\}, \\
& \mathcal{U}^{p}(\Omega):=\left\{u \in G S B V(\Omega) \cap L^{p}(\Omega): \nabla u \in L^{2}\left(\Omega, \mathbb{R}^{2}\right) \text { and } \mathcal{H}^{1}\left(S_{u}\right)<+\infty\right\},
\end{aligned}
$$

where $p \in[1,+\infty)$. Note that $\mathcal{U}^{p}(\Omega) \cap L^{\infty}(\Omega) \subseteq S B V^{2}(\Omega)$.

We say that a sequence $u_{k}$ in $S B V^{2}(\Omega)$ converges weakly to some $u \in S B V^{2}(\Omega)$, and we write $u_{k} \rightarrow u$ in $S B V^{2}(\Omega)$ if

$$
\left\{\begin{array}{l}
u_{k} \text { is bounded in } L^{\infty}(\Omega), \\
u_{k} \rightarrow u \text { strongly in } L^{1}(\Omega), \\
\nabla u_{k} \rightarrow \nabla u \text { weakly in } L^{2}\left(\Omega, \mathbb{R}^{2}\right), \\
\mathcal{H}^{1}\left(S_{u_{k}}\right) \text { is bounded. }
\end{array}\right.
$$


We define the Mumford-Shah functional $M S: L^{p}(\Omega) \rightarrow[0,+\infty]$ as

$$
M S(u):= \begin{cases}\int_{\Omega}|\nabla u(x)|^{2} d x+\mathcal{H}^{1}\left(S_{u}\right) & \text { if } u \in \mathcal{U}^{p}(\Omega), \\ +\infty & \text { otherwise. }\end{cases}
$$

We make explicit the dependence on the domain $\Omega$ with the notation $M S(\cdot, \Omega)$.

Remark 2.1. Let $u_{k}$ be a sequence in $\mathcal{U}^{p}(\Omega)$ such that $u_{k} \rightarrow u$ strongly in $L^{p}(\Omega)$ and $M S\left(u_{k}\right)$ is bounded. By the compactness result in $G S B V(\Omega)$ stated in [1, Theorem 4.36], it follows that $u \in \mathcal{U}^{p}(\Omega), \nabla u_{k} \rightarrow \nabla u$ weakly in $L^{2}\left(\Omega, \mathbb{R}^{2}\right)$, and $\lim _{\inf _{k}} M S\left(u_{k}\right) \geq M S(u)$.

Finally, we consider the family of functionals $F_{\varepsilon}: L^{p}(\Omega) \rightarrow[0,+\infty]$ defined by

$$
F_{\varepsilon}(u):= \begin{cases}\int_{\Omega}|\nabla u(x)|^{2} d x+\mathcal{H}^{1}\left(S_{u}\right) & \text { if } u \in \mathcal{U}^{p}(\Omega) \text { and } S_{u} \subseteq \Omega_{\varepsilon} \\ +\infty & \text { otherwise }\end{cases}
$$

where $\Omega_{\varepsilon}:=\Omega \cap \varepsilon\left([\delta, 1-\delta]^{2}+\mathbb{Z}^{2}\right)$ and $\delta=\delta(\varepsilon) \in(0,1 / 2)$.

The goal of this paper is to analyze the $\Gamma$-limit, as $\varepsilon \rightarrow 0^{+}$, of $F_{\varepsilon}$ in the space $L^{p}(\Omega)$ endowed with the strong topology. We refer to [4] for the definition and properties of $\Gamma$-convergence. In Theorem 4.1 and Theorem 5.1 we show that the $\Gamma$-limit depends on $\vartheta:=\lim _{\varepsilon} \delta(\varepsilon) / \varepsilon$.

Throughout the paper the characteristic function $\chi_{A}$ of a set $A$ is defined by $\chi_{A}(x)=1$ for $x \in A$ and $\chi_{A}(x)=0$ for $x \notin A$. The Lebesgue measure on $\mathbb{R}^{2}$ (resp., $\mathbb{R}$ ) is denoted by $\mathcal{L}^{2}\left(\right.$ resp., $\left.\mathcal{L}^{1}\right)$.

\section{A LOWER BOUnd estimate}

The aim of this section is to provide a lower bound for the $\Gamma$-limit of $F_{\varepsilon}$ on the characteristic functions of half-spaces, when the domain $\Omega$ is the unit square $Q:=(-1 / 2,1 / 2)^{2}$. We will use this estimate in Section 4 for the perfectly elastic case.

Theorem 3.1. Assume $\Omega=Q$. Given $t \geq 0$ and $\nu \in \mathbb{R}^{2}$, with $|\nu|=1$, we define the function $u_{t, \nu}: Q \rightarrow \mathbb{R}$ by

$$
u_{t, \nu}:=t \chi_{\{x \in Q: x \cdot \nu \leq 0\}} .
$$

Then, for any sequence $\varepsilon_{k} \rightarrow 0^{+}$and any sequence $u_{k}$ in $L^{p}(Q)$ such that $u_{k} \rightarrow u_{t, \nu}$ strongly in $L^{p}(Q)$, we have the estimate

$$
\liminf _{k \rightarrow+\infty} F_{\varepsilon_{k}}\left(u_{k}\right) \geq c_{0} \sqrt{\vartheta} t
$$

where $c_{0}>0$ is an absolute constant.

In the proof of Theorem 3.1 we will use the following two lemmas. The first is a wellknown trick that allows us to modify a sequence $u_{k}$ keeping the limit. The second is an ad hoc adaptation of the idea used in [11, Subsection 4.2]. For the readers convenience, we prefer to present here a simplified proof with suitable modifications.

Lemma 3.2. Let $B$ be a Borel subset of $(0,1)^{2}$, with $\mathcal{L}^{2}(B)>0$, and let $\varepsilon_{k} \rightarrow 0^{+}$. Given two sequences $u_{k}$ and $v_{k}$ in $L^{1}(\Omega)$ such that $u_{k} \rightarrow u$ and $v_{k} \rightarrow v$ strongly in $L^{1}(\Omega)$, suppose that

Then $u=v \quad \mathcal{L}^{2}$-a.e. on $\Omega$.

$$
u_{k}=v_{k} \quad \text { in } \quad B_{k}:=\Omega \cap \varepsilon_{k}\left(B+\mathbb{Z}^{2}\right) .
$$


Proof. By the Riemann-Lebesgue lemma, $\chi_{B_{h}}$ tends to $b:=\mathcal{L}^{2}(B)$ weakly* in $L^{\infty}(\Omega)$. Since the sequence $\left(u_{k}-v_{k}\right) \chi_{B_{k}}$ converges to $(u-v) b$ weakly in $L^{1}(\Omega)$, and $\left(u_{k}-v_{k}\right) \chi_{B_{k}}=0$ $\mathcal{L}^{2}$-a.e. on $\Omega$, we deduce that $(u-v) b=0 \quad \mathcal{L}^{2}$-a.e. on $\Omega$. Since $b>0$, we conclude that $u=v \quad \mathcal{L}^{2}$-a.e. on $\Omega$.

Lemma 3.3 (patching lemma). Let $Q:=(-1 / 2,1 / 2)^{2}$. For any $u \in S B V^{2}(Q) \cap L^{\infty}(Q)$, with $\mathcal{H}^{1}\left(S_{u}\right) \leq 1 / 28$, there exists $v \in S B V^{2}(Q) \cap L^{\infty}(Q)$ such that

(i) $v$ is constant in $(-1 / 2,1 / 2) \times(-2 / 7,2 / 7)$,

(ii) $v=u$ in $(-1 / 2,1 / 2) \times[(-1 / 2,-3 / 7) \cup(3 / 7,1 / 2)]$,

(iii) $\|\nabla v\|_{L^{2}\left(Q, \mathbb{R}^{2}\right)} \leq c_{1}\|\nabla u\|_{L^{2}\left(Q, \mathbb{R}^{2}\right)}$ for an absolute constant $c_{1}>0$,

(iv) $\|v\|_{L^{\infty}(Q)} \leq\|u\|_{L^{\infty}(Q)}$,

(v) $S_{v} \subseteq S_{u}$,

(vi) if $u(-1 / 2, \cdot)=u(1 / 2, \cdot)$ on $(-1 / 2,1 / 2)$, the same property holds for $v$.

Proof. A similar result in Sobolev spaces can be obtained by taking the constant in (i) equal to the mean value $m$ of $u$ on $Q$, and defining $v:=\phi(u-m)+m$ for a suitable cutoff function $\phi$. Estimate (iii) follows from the Poincaré-Wirtinger inequality. The same technique cannot be applied directly in the $S B V$ setting, because the Poincaré-Wirtinger inequality provided by De Giorgi, Carriero and Leaci [10, Theorem 3.1] requires a truncation of $u$. If we truncate on the whole square $Q$, condition (ii) may not be satisfied. On the other hand, if we truncate on only an intermediate strip, we may introduce additional cracks, in contrast with condition (v).

Therefore, the first step in our proof is a careful choice of the truncation strip, based on the behavior of the one-dimensional slices. Then the estimate on the oscillation will be obtained from the Poincaré-Wirtinger inequality for Sobolev functions in dimension one.

Step 1: Truncation. Since we are going to use the slicing procedure, we need to fix the precise representative $u^{*}$ of $u$ as defined in [1, Definition 3.63 and Corollary 3.80]. We make use of the following notation:

- $L_{r}:=(-1 / 2,1 / 2) \times\{r\}$ and $L^{r}:=\{r\} \times(-1 / 2,1 / 2), r \in \mathbb{R}$,

- $I:=\left\{r \in(5 / 14,3 / 7): L_{r} \cap S_{u^{*}}=\varnothing\right\}$,

- $J:=\left\{r \in(-3 / 7,-5 / 14): L_{r} \cap S_{u^{*}}=\varnothing\right\}$,

- $H:=\left\{r \in(-1 / 2,1 / 2): L^{r} \cap S_{u^{*}}=\varnothing\right\}$,

- $\operatorname{osc}_{r}\left(u^{*}\right):=\sup _{L_{r}} u^{*}-\inf _{L_{r}} u^{*}$ the oscillation of $u^{*}$ along $L_{r}, r \in(-1 / 2,1 / 2)$,

- $\operatorname{osc}^{r}\left(u^{*}\right):=\sup _{L^{r}} u^{*}-\inf _{L^{r}} u^{*}$ the oscillation of $u^{*}$ along $L^{r}, r \in(-1 / 2,1 / 2)$.

Since $\mathcal{H}^{1}\left(S_{u}\right) \leq 1 / 28$ we have $\mathcal{L}^{1}(I) \geq 1 / 28, \mathcal{L}^{1}(J) \geq 1 / 28$, and $\mathcal{L}^{1}(H) \geq 27 / 28$. Moreover, by [1, Theorems 3.28, 3.107, and 3.108], for $\mathcal{L}^{1}$-a.e. $r \in I$ the function $u^{*}(\cdot, r)$ is absolutely continuous with derivative given $\mathcal{L}^{1}$-a.e. by $\partial_{x_{1}} u^{*}(\cdot, r)$. Then

$$
\operatorname{osc}_{r}\left(u^{*}\right) \leq \int_{0}^{1}\left|\partial_{x_{1}} u^{*}\left(x_{1}, r\right)\right| d x_{1},
$$

and so, integrating over $I$, we obtain $\int_{I} \operatorname{osc}_{r}\left(u^{*}\right) d r \leq\|\nabla u\|_{L^{1}\left(Q, \mathbb{R}^{2}\right)}$. As $\mathcal{L}^{1}(I) \geq 1 / 28$, by the mean value theorem there exists $r_{1} \in(5 / 14,3 / 7)$ such that

$$
\operatorname{Osc}_{r_{1}}\left(u^{*}\right) \leq 28\|\nabla u\|_{L^{1}\left(Q, \mathbb{R}^{2}\right)} .
$$

Similarly, it is possible to prove that there exist $r_{2} \in(-3 / 7,-5 / 14)$ and $r_{3} \in(-1 / 2,1 / 2)$ such that $\operatorname{osc}_{r_{2}}\left(u^{*}\right) \leq 28\|\nabla u\|_{L^{1}\left(Q, \mathbb{R}^{2}\right)}$ and $\operatorname{osc}^{r_{3}}\left(u^{*}\right) \leq 2\|\nabla u\|_{L^{1}\left(Q, \mathbb{R}^{2}\right)}$. Since $L:=L_{r_{1}} \cup$ 


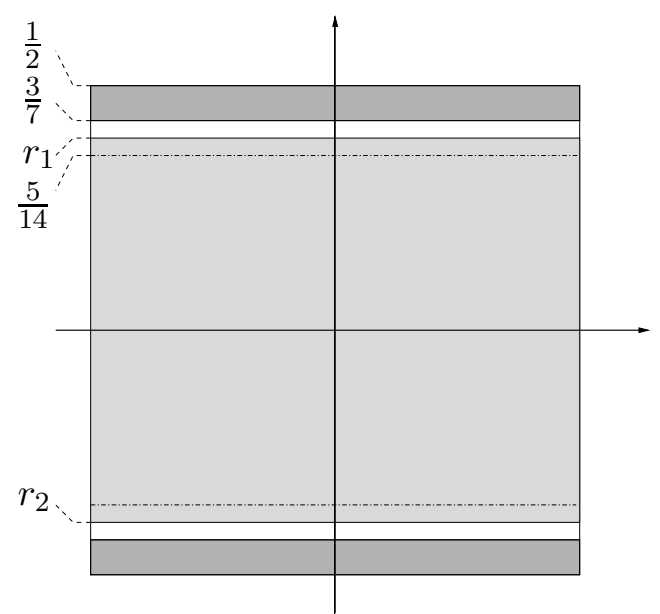

FIGURE 2

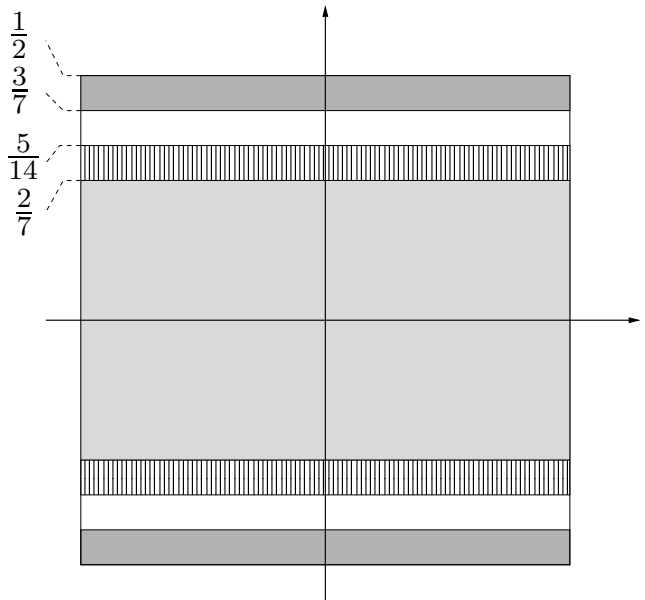

FIGURE 3

$L_{r_{2}} \cup L^{r_{3}}$ is connected, if $m_{u^{*}}:=\left(\sup _{L} u^{*}+\inf _{L} u^{*}\right) / 2$, we have that

$$
\left|u^{*}(x)-m_{u^{*}}\right| \leq c_{u^{*}} \text { for } \mathcal{H}^{1} \text {-a.e. } \quad x \in L,
$$

where $c_{u^{*}}:=30\|\nabla u\|_{L^{1}\left(Q, \mathbb{R}^{2}\right)}$.

In this way we can truncate the function $u^{*}$ on $(-1 / 2,1 / 2) \times\left[r_{2}, r_{1}\right]$ (the light gray part of Figure 2) without generating a new crack along $L_{r_{1}}$ and $L_{r_{2}}$ :

$$
w:= \begin{cases}{\left[u^{*} \wedge\left(m_{u^{*}}+c_{u^{*}}\right)\right] \vee\left(m_{u^{*}}-c_{u^{*}}\right)} & \text { in }(-1 / 2,1 / 2) \times\left[r_{2}, r_{1}\right], \\ u^{*} & \text { in }(-1 / 2,1 / 2) \times\left[\left(-1 / 2, r_{2}\right) \cup\left(r_{1}, 1 / 2\right)\right] .\end{cases}
$$

Obviously $w$ satisfies (ii)-(vi) and $\left|w(x)-m_{u^{*}}\right| \leq 30\|\nabla u\|_{L^{1}\left(Q, \mathbb{R}^{2}\right)}$ for $x_{2} \in(-5 / 14,5 / 14)$.

Step 2: Cut-Off. Let $\phi \in C^{1}((-1 / 2,1 / 2),[0,1])$ be a cut-off function such that

$$
\phi(t)= \begin{cases}0 & \text { if } t \in(-2 / 7,2 / 7), \\ 1 & \text { if } t \in(-1 / 2,-5 / 14) \cup(5 / 14,1 / 2),\end{cases}
$$

and $\left|\phi^{\prime}(t)\right| \leq 15$. If we define the function $v$ on $Q$ as $v(x):=\phi\left(x_{2}\right)\left[w(x)-m_{u^{*}}\right]+m_{u^{*}}$, by construction $v$ satisfies (i), (ii), and (iv)-(vi). In particular $v$ is constant on $(-1 / 2,1 / 2) \times$ $(-2 / 7,2 / 7)$ (the light gray part of Figure 3$)$.

Let $U:=(-1 / 2,1 / 2) \times[(-5 / 14,-2 / 7) \cup(2 / 7,5 / 14)]$. Since $\nabla v=\phi \nabla w+\left(w-m_{u^{*}}\right)\left(0, \phi^{\prime}\right)$ and $\left\|\phi^{\prime}\left(w-m_{u^{*}}\right)\right\|_{L^{\infty}(Q)} \leq 15 \sup _{U}\left|w-m_{u^{*}}\right| \leq 450\|\nabla u\|_{L^{1}\left(Q, \mathbb{R}^{2}\right)} \leq 450\|\nabla u\|_{L^{2}\left(Q, \mathbb{R}^{2}\right)}$, we have that $v$ satisfies also (iii): $\|\nabla v\|_{L^{2}\left(Q, \mathbb{R}^{2}\right)} \leq 451\|\nabla u\|_{L^{2}\left(Q, \mathbb{R}^{2}\right)}$.

Proof of Theorem 3.1. Let $\Theta:=\liminf _{k} F_{\varepsilon_{k}}\left(u_{k}\right)$. If $\Theta$ is finite, by extracting a subsequence we may assume that the liminf is a limit, that $u_{k} \in \mathcal{U}^{p}(Q)$, and that $M S\left(u_{k}\right)$ is bounded. By truncating $u_{k}$ between 0 and $t$, we may also have $u_{k} \in S B V^{2}(Q) \cap L^{\infty}(Q)$ and $\left\|u_{k}\right\|_{L^{\infty}(Q)}$ bounded.

The plan of the proof is the following. In the first step we construct a new sequence $u_{k}^{p e r}$, with $u_{k}^{\text {per }}\left(x_{1}+\varepsilon_{k}, x_{2}\right)=u_{k}^{\text {per }}\left(x_{1}, x_{2}\right)$, whose energy is controlled from above by $M S\left(u_{k}\right)$ and whose limit $u^{\text {per }}$ has the special form $u^{p e r}=t \chi_{(-1 / 2,1 / 2) \times(-1 / 2, a)}$ for some $a \in(-1 / 2,1 / 2)$. In the second step we regularize $u_{k}^{p e r}$ in the squares of size $\varepsilon_{k}$ where the jump set is very 
small with respect to $\varepsilon_{k}$. In this way we construct a new periodic sequence $u_{k}^{r e g}$, with the same limit $u^{p e r}$, whose energy is still controlled from above by $M S\left(u_{k}\right)$, and whose cracks are now concentrated only in a bounded number of horizontal strips of height $\varepsilon_{k}$. The special form of the functions $u_{k}^{r e g}$ and $u^{\text {per }}$ will allow us to prove estimate (3.1) for $u_{k}^{r e g}$, and the control of the energies will give (3.1) for $u_{k}$.

We assume initially that

$$
\varepsilon_{k}^{-1} \text { is an even integer. }
$$

Step 1: Symmetrization. With suitable ninety degree rotations, we can suppose that $\nu=(\cos \gamma, \sin \gamma)$ with $\gamma \in[\pi / 4,3 \pi / 4]$. We fix a small $\eta>0$ and we choose a sequence $\lambda_{k} \rightarrow+\infty$ such that

$$
\lambda_{k} \int_{Q}\left|u_{t, \nu}-u_{k}\right|^{p} d x \leq \eta .
$$

For any integer $j \in\left\{-\varepsilon_{k}^{-1}, \ldots, \varepsilon_{k}^{-1}-1\right\}$ we consider the functional

$$
F_{j}^{k}\left(v^{(1)}, v^{(2)}\right):=M S\left(v^{(2)}, X_{j}^{k}\right)+\lambda_{k} \int_{X_{j}^{k}}\left|v^{(1)}-v^{(2)}\right|^{p} d x,
$$

defined for $v^{(1)}, v^{(2)} \in S B V^{2}(Q)$, where $X_{j}^{k}$ denotes the vertical strip $\left(j \varepsilon_{k} / 2,(j+1) \varepsilon_{k} / 2\right) \times$ $(-1 / 2,1 / 2)$.

For any $k \in \mathbb{N}$ let $j_{k}$ be an integer such that

$$
F_{j_{k}}^{k}\left(u_{t, \nu}, u_{k}\right)=\min \left\{F_{j}^{k}\left(u_{t, \nu}, u_{k}\right): j \in\left\{-\varepsilon_{k}^{-1}, \ldots, \varepsilon_{k}^{-1}-1\right\}\right\},
$$

and let $u_{k}^{\text {per }}$ (resp., $w_{k}$ ) be the only extension of $\left.u_{k}\right|_{X_{j_{k}}^{k}}$ (resp., $\left.\left.u_{t, \nu}\right|_{X_{j_{k}}^{k}}\right)$ to $\mathbb{R} \times(-1 / 2,1 / 2)$ that is symmetric with respect to all vertical lines $x_{2}=j \varepsilon_{k} / 2$ with $j \in \mathbb{Z}$. Note that $S_{u_{k}^{p e r}} \subseteq \varepsilon_{k}\left(\left[\delta_{k}, 1-\delta_{k}\right]^{2}+\mathbb{Z}^{2}\right)$ and that

$$
u_{k}^{\text {per }}\left(x_{1}, x_{2}\right)=u_{k}^{\text {per }}\left(x_{1}+\varepsilon_{k}, x_{2}\right)
$$

for every $\left(x_{1}, x_{2}\right) \in \mathbb{R} \times(-1 / 2,1 / 2)$ and every $k \in \mathbb{N}$.

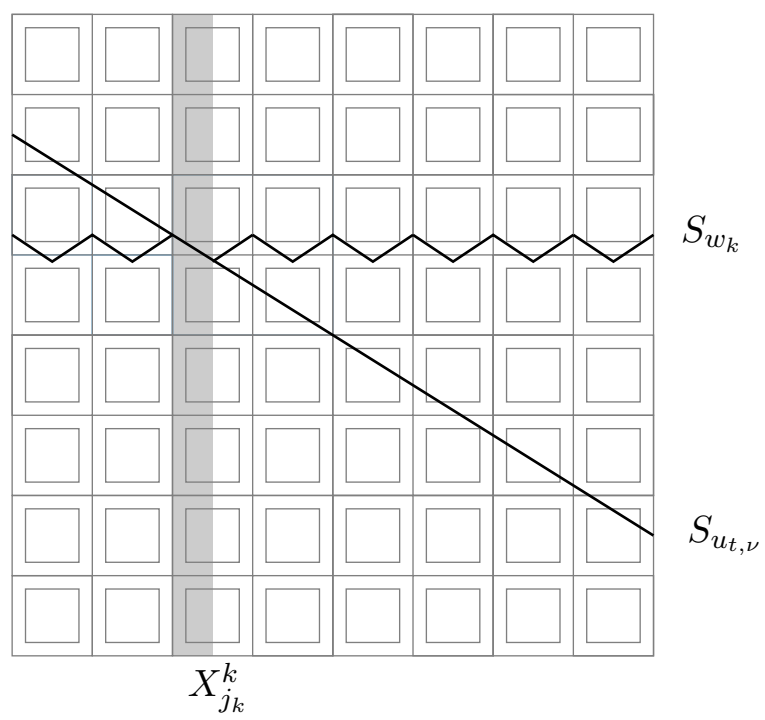

FigURE 4 
Obviously we have $M S\left(w_{k}, Q\right)=M S\left(u_{t, \nu}, Q\right)$. Moreover, from the minimality of $X_{j_{k}}^{k}$ and (3.3) we deduce that

$$
\begin{aligned}
& M S\left(u_{k}^{p e r}, Q\right)+\lambda_{k} \int_{Q}\left|w_{k}-u_{k}^{p e r}\right|^{p} d x=2 \varepsilon_{k}^{-1}\left[M S\left(u_{k}, X_{j_{k}}^{k}\right)+\lambda_{k} \int_{X_{j_{k}}^{k}}\left|u_{t, \nu}-u_{k}\right|^{p} d x\right] \\
& =2 \varepsilon_{k}^{-1} F_{j_{k}}^{k}\left(u_{t, \nu}, u_{k}\right) \leq \sum_{j=-\varepsilon_{k}^{-1}}^{\varepsilon_{k}^{-1}-1} F_{j}^{k}\left(u_{t, \nu}, u_{k}\right)=M S\left(u_{k}, Q\right)+\lambda_{k} \int_{Q}\left|u_{t, \nu}-u_{k}\right|^{p} d x \\
& \leq M S\left(u_{k}, Q\right)+\eta .
\end{aligned}
$$

Since $w_{k}$ and $u_{k}^{\text {per }}$ are also bounded in $L^{\infty}(Q)$, we can apply the compactness result in $S B V(Q)$ stated in [1, Theorem 4.8], obtaining the existence of $w$ and $u^{p e r}$ in $S B V^{2}(Q)$ such that (up to subsequences, not relabeled) $w_{k} \rightarrow w$ and $u_{k}^{p e r} \rightarrow u^{\text {per }}$ weakly in $S B V^{2}(Q)$. From (3.5) we deduce also that

$$
\sup _{k}\left\{\lambda_{k}\left\|w_{k}-u_{k}^{p e r}\right\|_{L^{p}(Q)}^{p}\right\}<+\infty .
$$

Since $\lambda_{k} \rightarrow+\infty$, we conclude that $w=u^{\text {per }}$. Now let $g_{k}:(-1 / 2,1 / 2) \rightarrow(-1 / 2,1 / 2)$ be the Lipschitz function on $(-1 / 2,1 / 2)$ whose graph is $S_{w_{k}}$ (see Figure 4$)$. The oscillation of $g_{k}$ is bounded by $\varepsilon_{k}|\cot \gamma| / 2$ and, therefore, up to a subsequence (not relabeled), $g_{k}$ converges uniformly to a certain constant $a$. If $\gamma \in(\pi / 4,3 \pi / 4)$, then $a \in(-1 / 2,1 / 2)$. Instead, if $\gamma=\pi / 4$ or $3 \pi / 4$, with the previous construction we could have $a= \pm 1 / 2$. To avoid this possibility, when $\gamma=\pi / 4$ or $3 \pi / 4$ we slightly change the construction of $u_{k}^{p e r}$. Recalling that $\varepsilon_{k}^{-1}$ is an even integer, we choose the vertical strips $X_{j}^{k}$ only in $(-1 / 4,1 / 4) \times(-1 / 2,1 / 2)$ so that $a \in[-1 / 4,1 / 4]$. In this case $(3.5)$ has to be modified and we get

$$
M S\left(u_{k}^{p e r}, Q\right)+\lambda_{k} \int_{Q}\left|w_{k}-u_{k}^{p e r}\right|^{p} d x \leq 2 M S\left(u_{k}, Q\right)+2 \eta .
$$

Given $\xi>0$, for $k$ sufficiently large

$$
w_{k}(x)= \begin{cases}0 & \text { if } x \in(-1 / 2,1 / 2) \times[a+\xi, 1 / 2), \\ t & \text { if } x \in(-1 / 2,1 / 2) \times(-1 / 2, a-\xi],\end{cases}
$$

therefore $u^{\text {per }}=t \chi_{(-1 / 2,1 / 2) \times(-1 / 2, a)}$.

Step 2: Regularization. We smooth out $u_{k}^{\text {per }}$ on those squares where $S_{u_{k}^{\text {per }}}$ is small by using Lemma 3.3. For any $i, j \in I_{k}:=\left\{-\varepsilon_{k}^{-1} / 2, \ldots, \varepsilon_{k}^{-1} / 2-1\right\}$ we define the following sets:

- $Q_{i, j}:=\varepsilon_{k}(0,1)^{2}+\left(i \varepsilon_{k}, j \varepsilon_{k}\right)$,

- $Q_{i, j}^{\prime}:=\varepsilon_{k}((0,1) \times(1 / 14,13 / 14))+\left(i \varepsilon_{k}, j \varepsilon_{k}\right)$,

- $Q_{i, j}^{\prime \prime}:=\varepsilon_{k}((0,1) \times(3 / 14,11 / 14))+\left(i \varepsilon_{k}, j \varepsilon_{k}\right)$,

- $T_{j}:=(-1 / 2,1 / 2) \times\left(j \varepsilon_{k},(j+1) \varepsilon_{k}\right)$.

Note that by the periodicity property (3.4), if

$$
\mathcal{H}^{1}\left(S_{u_{k}^{\text {per }}} \cap Q_{i, j}\right)<\varepsilon_{k} / 56
$$

for a certain $(i, j)$, then $\mathcal{H}^{1}\left(S_{u_{k}^{p e r}} \cap Q_{h, j}\right)<\varepsilon_{k} / 56$ for all $h \in I_{k}$, i.e., condition (3.7) is satisfied by all squares in the strip $T_{j}$. Let $J_{k}$ be the set of the indices $j \in\left\{1-\varepsilon_{k}^{-1}, \ldots, \varepsilon_{k}^{-1}-\right.$ 
$2\}$ such that all squares in the strips $T_{j-1}, T_{j}$ and $T_{j+1}$ satisfy (3.7). Moreover, let $L_{k}^{1}, \ldots, L_{k}^{m_{k}}$ be the connected components of $L_{k}:=\operatorname{int}\left(\bigcup_{j \in J_{k}} \bar{T}_{j}\right)$. We enumerate these blocks from bottom to top.

Thanks to Lemma 3.3, together with a rescaling argument, in every square $Q_{i, j}$ satisfying (3.7) we can patch $u_{k}^{p e r}$ on $Q_{i, j}^{\prime}$ so that it becomes smooth (because constant) in $Q_{i, j}^{\prime \prime}$ (see Figure 5). We remark in particular that by keeping the periodicity condition (3.4), we do not create new cracks along the vertical boundary of the squares $Q_{i, j}$. Moreover, since after this patching the Mumford-Shah energy remains bounded, by the compactness result in $S B V(Q)$ stated in [1, Theorem 4.8] we may assume that the modification of $u_{k}^{p e r}$ still converges weakly in $S B V^{2}(Q)$. Since the sequence is unchanged on $Q_{i, j} \backslash Q_{i, j}^{\prime}$, thanks to Lemma 3.2 the limit remains $u^{\text {per }}$.

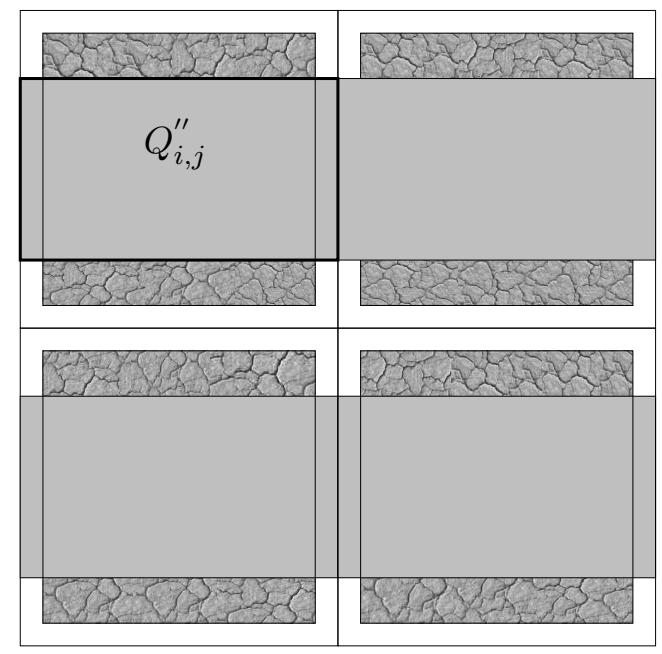

FigURE 5

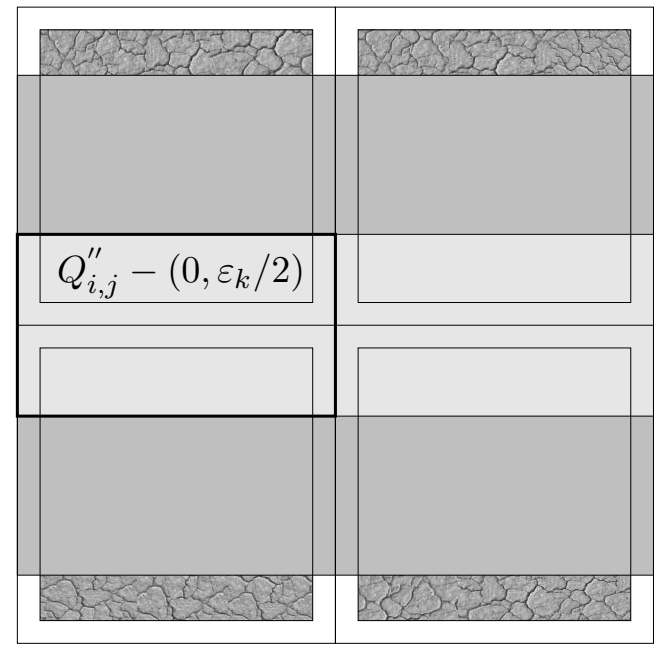

FIGURE 6

For $j \in J_{k}$, since $\mathcal{H}^{1}\left(S_{u_{k}^{p e r}} \cap\left(Q_{i, j} \pm\left(0, \varepsilon_{k} / 2\right)\right)\right)<\varepsilon_{k} / 28$, we can use Lemma 3.3 also in $Q_{i, j} \pm\left(0, \varepsilon_{k} / 2\right)$ to further modify $u_{k}^{p e r}$, so that the modified function has no cracks in $T_{j}$ and is constant on the light gray strips of Figures 6 and 7:

$$
\bigcup_{i \in I_{k}} \bigcup_{j \in J_{k}} Q_{i, j}^{\prime \prime} \pm\left(0, \varepsilon_{k} / 2\right)
$$

Let $u_{k}^{r e g}$ be the sequence obtained by regularizing $u_{k}^{\text {per }}$ through the above two modifications. By construction $u_{k}^{r e g}$ converges weakly to $u^{p e r}$ in $S B V^{2}(Q)$,

$$
\int_{Q}\left|\nabla u_{k}^{r e g}(x)\right|^{2} d x \leq c_{1}^{4} \int_{Q}\left|\nabla u_{k}^{p e r}(x)\right|^{2} d x, \quad \text { and } \quad S_{u_{k}^{r e g}} \subseteq S_{u_{k}^{p e r}},
$$

where $c_{1}$ is the constant in Lemma 3.3. Moreover, the function $u_{k}^{r e g}$ belongs to $H^{1}\left(L_{k}\right)$ and it is constant along the horizontal boundary of each block $L_{k}^{h}$ for $h \in\left\{1, \ldots, m_{k}\right\}$.

Now let $v_{k} \in H^{1}(Q)$ be a function with zero average such that $\nabla v_{k}=\nabla u_{k}^{r e g}$ on $L_{k}$ and $\nabla v_{k}=0$ otherwise. Up to a subsequence, $v_{k}$ converges weakly to a certain $v$ in $H^{1}(Q)$. Since each $v_{k}$ is $\varepsilon_{k}$-periodic in $x_{1}$, the function $v$ depends only on $x_{2}$. Let us define $\tilde{u}_{k}:=u_{k}^{r e g}-v_{k}$. By construction $\tilde{u}_{k}$ is constant on each block $L_{k}^{h}$ for $h \in\left\{1, \ldots, m_{k}\right\}$, and 


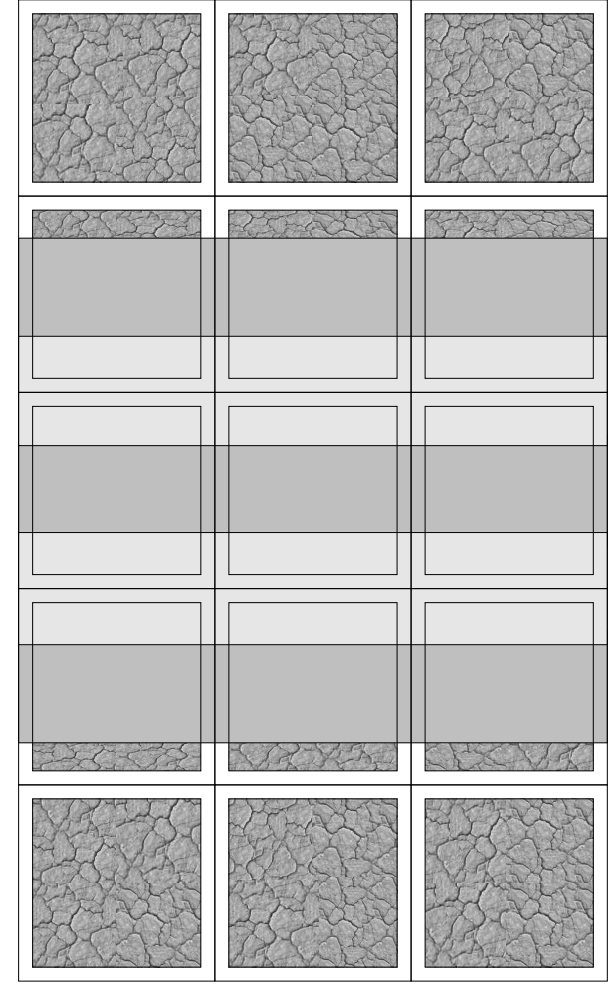

Figure 7

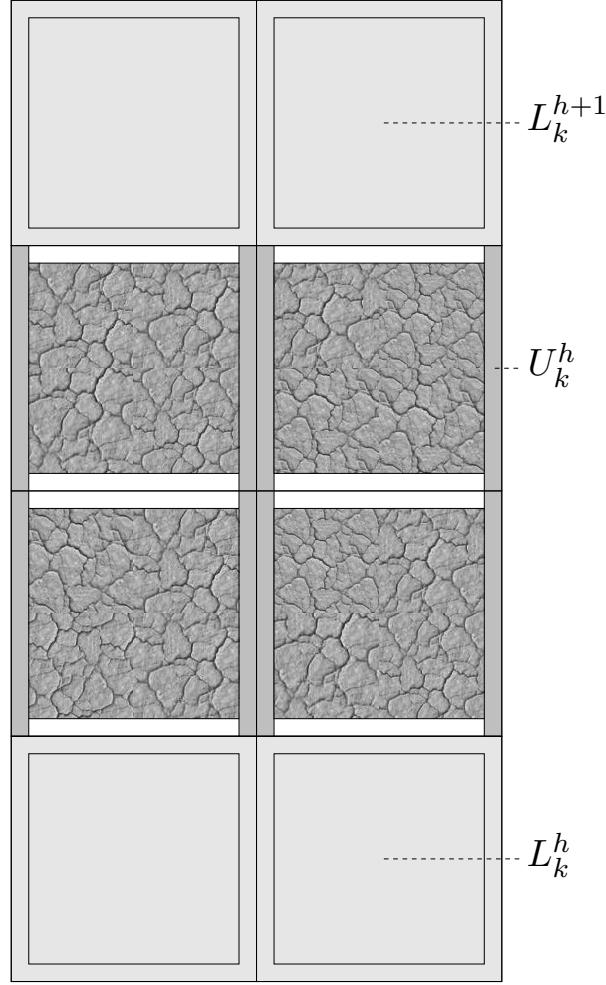

FIGURE 8

$D \tilde{u}_{k}=D u_{k}^{r e g}$ otherwise, so that

$$
\left|\nabla \tilde{u}_{k}\right| \leq\left|\nabla u_{k}^{r e g}\right| \quad \mathcal{L}^{2} \text {-a.e. on } Q \quad \text { and } \quad S_{\tilde{u}_{k}}=S_{u_{k}^{r e g}} .
$$

Moreover, $\tilde{u}_{k}$ converges weakly to $\tilde{u}:=u^{p e r}-v$ in $S B V^{2}(Q), S_{\tilde{u}}=S_{u^{p e r}}=(-1 / 2,1 / 2) \times$ $\{a\}$, and $[\tilde{u}]=t$ on $S_{\tilde{u}}$.

Let $n_{k}$ be the number of strips $T_{j}$ whose squares $Q_{i, j}$ do not satisfy (3.7). Since on these strips $u_{k}^{r e g}=u_{k}^{p e r}$, resulting in $\tilde{u}_{k}$ and $u_{k}^{p e r}$ having the same cracks, we trivially have

$$
\mathcal{H}^{1}\left(S_{\tilde{u}_{k}}\right) \geq n_{k} / 56
$$

In order to complete the proof we need also to estimate $\int_{Q}\left|\nabla \tilde{u}_{k}(x)\right|^{2} d x$. We start by looking over the oscillation of $\tilde{u}_{k}$ on $L_{k}$. Let us observe that a strip $T_{j}$ is not included in $L_{k}$ if and only if it is contiguous to a strip of squares $Q_{i, j}$ not satisfying (3.7). Therefore, by (3.10) it follows that

$$
\mathcal{L}^{2}\left(Q \backslash L_{k}\right) \leq 3 n_{k} \varepsilon_{k} \leq 168 \varepsilon_{k} \mathcal{H}^{1}\left(S_{\tilde{u}_{k}}\right) .
$$

Since $\mathcal{H}^{1}\left(S_{\tilde{u}_{k}}\right)$ is bounded, the previous inequality implies that $\mathcal{L}^{2}\left(Q \backslash L_{k}\right) \rightarrow 0$, i.e., the blocks $L_{k}^{1}, \ldots, L_{k}^{m_{k}}$ tend to invade the whole domain $Q$.

Let $b_{k}^{h}$ be the constant value assumed by $\tilde{u}_{k}$ on $L_{k}^{h}$. The function $\tilde{u}$ is continuous in $(-1 / 2,1 / 2) \times(-1 / 2, a)$ and $(-1 / 2,1 / 2) \times(a, 1 / 2)$ (because it is $H^{1}$ and depends only on $\left.x_{2}\right)$, and $[\tilde{u}]=t$ on $(-1 / 2,1 / 2) \times\{a\}$. Therefore, there exists $\tau>0$ such that the difference between the infimum of $\tilde{u}$ in $(-1 / 2,1 / 2) \times(a-\tau, a)$ and the supremum in $(-1 / 2,1 / 2) \times$ $(a, a+\tau)$ is greater than $t / 2$. Since $\tilde{u}_{k} \rightarrow \tilde{u}$ strongly in $L^{1}(Q)$ and $\mathcal{L}^{2}\left(Q \backslash L_{k}\right) \rightarrow 0$, we 
have

$$
O_{k}:=\max \left\{b_{k}^{1}, \ldots, b_{k}^{m_{k}}\right\}-\min \left\{b_{k}^{1}, \ldots, b_{k}^{m_{k}}\right\} \geq t / 2
$$

for $k$ large enough.

Now we need some additional notation:

- $d_{k}^{h}$ is the distance between $L_{k}^{h}$ and $L_{k}^{h+1}$;

- $U_{k}^{h}$ is the set composed of the vertical frames of the squares belonging to the strips $T_{j}$ lying between $L_{k}^{h}$ and $L_{k}^{h+1}$ (the dark gray vertical zones of Figure 8, see (3.13) below);

- $d_{k}:=\sum_{h=1}^{m_{k}} d_{k}^{h}$ and $U_{k}:=\bigcup_{h=1}^{m_{k}} U_{k}^{h}$.

In formulas, if $L_{k}^{h}=(-1 / 2,1 / 2) \times\left(l_{1}, l_{2}\right)$ and $L_{k}^{h+1}=(-1 / 2,1 / 2) \times\left(l_{3}, l_{4}\right)$, then $d_{k}^{h}:=l_{3}-l_{2}$ and

$$
U_{k}^{h}:=\bigcup_{i \in I_{k}}\left[\varepsilon_{k}\left(\left[0, \delta_{k}\right) \cup\left(1-\delta_{k}, 1\right]\right)+i \varepsilon_{k}\right] \times\left[l_{2}, l_{3}\right],
$$

so that $\mathcal{L}^{2}\left(U_{k}^{h}\right)=2 d_{k}^{h} \delta_{k}$.

On each set $U_{k}^{h}$ we compare $\tilde{u}_{k}$ with the affine function with the same boundary conditions on $\partial U_{k}^{h} \cap\left(\partial L_{k}^{h} \cup \partial L_{k}^{h+1}\right)$, which provides the lowest energy on $U_{k}^{h}$, and we get

$$
\int_{U_{k}}\left|\nabla \tilde{u}_{k}(x)\right|^{2} d x \geq \sum_{h=1}^{m_{k}} \mathcal{L}^{2}\left(U_{k}^{h}\right)\left|\frac{b_{k}^{h+1}-b_{k}^{h}}{d_{k}^{h}}\right|^{2}=2 \delta_{k} \sum_{h=1}^{m_{k}} \frac{\left|b_{k}^{h+1}-b_{k}^{h}\right|^{2}}{d_{k}^{h}} .
$$

By the Schwarz inequality (applied to the vectors with components $\left|b_{k}^{h+1}-b_{k}^{h}\right| /\left(d_{k}^{h}\right)^{1 / 2}$ and $\left(d_{k}^{h}\right)^{1 / 2}$ ) we have

$$
\sum_{h=1}^{m_{k}} \frac{\left|b_{k}^{h+1}-b_{k}^{h}\right|^{2}}{d_{k}^{h}} \geq \frac{1}{d_{k}}\left(\sum_{h=1}^{m_{k}}\left|b_{k}^{h+1}-b_{k}^{h}\right|\right)^{2} \geq \frac{O_{k}^{2}}{d_{k}},
$$

where $O_{k}$ is defined in (3.12). Since $d_{k} \leq 3 n_{k} \varepsilon_{k}$ by (3.11), we obtain from (3.14) and (3.15)

$$
\int_{U_{k}}\left|\nabla \tilde{u}_{k}(x)\right|^{2} d x \geq \frac{2 \delta_{k} O_{k}^{2}}{3 n_{k} \varepsilon_{k}} .
$$

Finally, combining (3.5), (3.6), (3.8)-(3.10), (3.12), and (3.16) we get

$$
\begin{aligned}
2(\Theta+\eta) & \geq \liminf _{k \rightarrow+\infty} M S\left(u_{k}^{\text {per }}, Q\right) \geq \liminf _{k \rightarrow+\infty}\left(\frac{1}{c_{1}^{4}} \int_{Q}\left|\nabla u_{k}^{r e g}(x)\right|^{2} d x+\mathcal{H}^{1}\left(S_{u_{k}^{r e g}}\right)\right) \\
& \geq \liminf _{k \rightarrow+\infty}\left(\frac{1}{c_{1}^{4}} \int_{Q}\left|\nabla \tilde{u}_{k}(x)\right|^{2} d x+\mathcal{H}^{1}\left(S_{\tilde{u}_{k}}\right)\right) \geq \liminf _{k \rightarrow+\infty}\left(\frac{\delta_{k} t^{2}}{6 c_{1}^{4} n_{k} \varepsilon_{k}}+\frac{n_{k}}{56}\right) \\
& \geq \liminf _{k \rightarrow+\infty} \sqrt{\frac{\delta_{k}}{42 \varepsilon_{k}}} \frac{t}{c_{1}^{2}} \geq \frac{\sqrt{\vartheta} t}{7 c_{1}^{2}},
\end{aligned}
$$

where the last inequality is obtained by minimizing with respect to $n_{k}$. Being $\eta>0$ arbitrary, we obtain (3.1).

In order to remove assumption (3.2) we consider, for $k \in \mathbb{N}$, the largest even integer $m_{k}$ such that $m_{k} \leq \varepsilon_{k}^{-1}$. Let $s_{k}:=m_{k} \varepsilon_{k}$. We define $\hat{u}_{k}: Q \rightarrow \mathbb{R}$ by

$$
\hat{u}_{k}(x):=u_{k}\left(s_{k} x\right) / \sqrt{s_{k}} \text {. }
$$


Since $s_{k}$ converges to 1 , we have that $\hat{u}_{k}$ tends to $u_{t, \nu}$ strongly in $L^{p}(Q)$. Moreover, $S_{\hat{u}_{k}} \subseteq m_{k}^{-1}\left(\left[\delta_{k}, 1-\delta_{k}\right]^{2}+\mathbb{Z}^{2}\right)$ and $\lim _{k} \delta_{k} m_{k}=\vartheta$. By applying the previous result we have

$$
\liminf _{k \rightarrow+\infty} F\left(u_{k}\right) \geq \liminf _{k \rightarrow+\infty} s_{k} M S\left(\hat{u}_{k}\right) \geq c_{0} \sqrt{\vartheta} t .
$$

Remark 3.4. Given a sequence $\varepsilon_{k} \rightarrow 0^{+}$, by a well-known compactness argument (see [4, Proposition 2.14]), there exists a subsequence $\varepsilon_{k_{j}}$ such that $F_{\varepsilon_{k_{j}}} \Gamma$-converges to some functional $F: L^{p}(Q) \rightarrow[0,+\infty]$. When $\vartheta \in(0,+\infty)$, it is proved in [8] that the functional $F$ admits an integral representation: there exists a Borel function $g:[0,+\infty) \times \mathbb{R}^{2} \rightarrow$ $[0,+\infty)$ such that

$$
F(u):= \begin{cases}\int_{Q}|\nabla u(x)|^{2} d x+\int_{S_{u}} g\left([u](x), \nu_{u}(x)\right) d \mathcal{H}^{1}(x) & \text { if } u \in \mathcal{U}^{p}(Q), \\ +\infty & \text { otherwise. }\end{cases}
$$

Estimate (3.1) shows that $g(t, \nu)$ cannot depend only on $\nu$. Indeed, fix $\nu \neq 0$ and let $l$ be the length of the segment $\{x \in Q: x \cdot \nu=0\}$, which coincides with the jump set of the functions $u_{t, \nu}$ considered in Theorem 3.1. Then (3.1) and (3.17) give

$$
g(t, \nu) l=F\left(u_{t, \nu}\right) \geq c_{0} \sqrt{\vartheta} t .
$$

This shows that we cannot have $g(t, \nu)=g(1, \nu)$ for every $t$.

\section{The PERFECTLY ELASTiC CASE}

In this section we prove the following result.

Theorem 4.1. Let $F_{\varepsilon}$ be defined as in (2.1). If $\vartheta=+\infty$, then $F_{\varepsilon} \Gamma$-converges to

$$
F_{\text {hom }}(u):= \begin{cases}\int_{\Omega}|\nabla u(x)|^{2} d x & \text { if } u \in H^{1}(\Omega), \\ +\infty & \text { otherwise, }\end{cases}
$$

as $\varepsilon \rightarrow 0^{+}$.

Proof. Let us fix $\varepsilon_{k} \rightarrow 0^{+}$. As $\Omega$ has Lipschitz boundary, if $u \in H^{1}(\Omega)$, then $u \in L^{p}(\Omega)$. In particular $u \in \mathcal{U}^{p}(\Omega)$ and $F_{\varepsilon_{k}}(u)=F_{\text {hom }}(u)$ for any $k$. This provides the limsup inequality with recovery sequence defined by $u_{k}:=u$.

To prove the liminf inequality we consider a sequence $u_{k}$ in $\mathcal{U}^{p}(\Omega)$ such that $u_{k}$ tends to $u$ strongly in $L^{p}(\Omega)$ and

$$
\sup _{k} F_{\varepsilon_{k}}\left(u_{k}\right)=: A<+\infty \text {. }
$$

By Remark 2.1, $u \in \mathcal{U}^{p}(\Omega), \nabla u_{k} \rightarrow \nabla u$ weakly in $L^{2}\left(\Omega, \mathbb{R}^{2}\right)$ and

$$
\liminf _{k \rightarrow+\infty} F_{\varepsilon_{k}}\left(u_{k}\right) \geq \int_{\Omega}|\nabla u(x)|^{2} d x+\mathcal{H}^{1}\left(S_{u}\right) .
$$

The liminf inequality is, therefore, proved when showing that $u \in H^{1}(\Omega)$, i.e., $\mathcal{H}^{1}\left(S_{u}\right)=0$.

We proceed by contradiction, assuming that

$$
\mathcal{H}^{1}\left(S_{u}\right)>0 .
$$

The idea is to carry out a blow up around a suitable jump point of $u$, so that we can apply Theorem 3.1. 
Since the sequence of measures $\mathcal{H}^{1}\left\llcorner S_{u_{k}}\right.$ is bounded by (4.1), there exists a subsequence (not relabeled) such that

$$
\mu_{k}:=\mathcal{H}^{1}\left\llcorner S_{u_{k}} \rightarrow \mu \quad\right. \text { weakly* in the sense of measures }
$$

for some nonnegative Radon measure $\mu$ with $\mu(\Omega)<+\infty$. For any $x \in \Omega$ we define

$$
l(x):=\limsup _{r \rightarrow 0^{+}} \frac{\mu\left(B_{r}(x)\right)}{r} .
$$

By applying $\left[1\right.$, Theorem 2.56], if $l(x)=+\infty$ on a Borel set $B$ with $\mathcal{H}^{1}(B)>0$, then necessarily $\mu(B)=+\infty$, in contradiction with $\mu(\Omega)<+\infty$. Therefore

$$
l(x)<+\infty \text { for } \mathcal{H}^{1} \text {-a.e. } x \in \Omega .
$$

Given $x \in S_{u}$ and $r>0$, with $B_{r}(x) \subseteq \Omega$, we define the following functions on $B_{1}(0)$ :

- $u_{k}^{r}(y):=u_{k}(x+r y)$,

- $u^{r}(y):=u(x+r y)$,

- $\tilde{u}:=\chi_{\left\{y \in B_{1}(0): y \cdot \nu_{u}(x) \leq 0\right\}}$.

By a change of variables we deduce that $u_{k}^{r} \rightarrow u^{r}$ strongly in $L^{p}\left(B_{1}(0)\right)$ as $k \rightarrow+\infty$. Moreover (see [1, Remark 3.72]), for $\mathcal{H}^{1}$-a.e. $x \in S_{u}$ we have that

$$
u^{r} \rightarrow \tilde{u} \text { strongly in } L^{p}\left(B_{1}(0)\right) \text { as } r \rightarrow 0^{+} .
$$

If (4.2) holds, by (4.4) we can choose $x \in S_{u}$ such that (4.5) holds and $l(x)<+\infty$.

Let $r_{h} \rightarrow 0^{+}$be such that $\mu\left(\partial B_{r_{h}}(x)\right)=0$ and $\mu\left(B_{r_{h}}(x)\right) / r_{h} \rightarrow l(x)$ as $h \rightarrow+\infty$. By (4.1) there exists a subsequence (not relabeled) such that for every $h$

$$
\lim _{k \rightarrow+\infty} M S\left(u_{k}^{r_{h}}, B_{1}(0)\right)=A_{h}+\frac{\mu\left(B_{r_{h}}(x)\right)}{r_{h}}
$$

for some constant $A_{h} \leq A$. Passing to a subsequence, we may assume that there exists a constant $\widetilde{A}<+\infty$ such that $A_{h} \rightarrow \widetilde{A}$ as $h \rightarrow+\infty$. Setting $\delta_{k}:=\delta\left(\varepsilon_{k}\right)$, we can apply a diagonal argument (see, e.g., [2, Corollary 1.18]) to the double indexed sequence $\left(u_{k}^{r_{h}}, M S\left(u_{k}^{r_{h}}, B_{1}(0)\right), \frac{\varepsilon_{k}}{\delta_{k} r_{h}}\right)$, and we find a sequence $h_{k} \rightarrow+\infty$ such that

$$
u_{k}^{r_{h_{k}}} \stackrel{L^{p}}{\longrightarrow} \tilde{u}, \quad M S\left(u_{k}^{r_{h_{k}}}, B_{1}(0)\right) \rightarrow \widetilde{A}+l(x), \quad \text { and } \quad \frac{\varepsilon_{k}}{\delta_{k} r_{h_{k}}} \rightarrow 0^{+} .
$$

Note that the last condition also implies that $\tilde{\varepsilon}_{k}:=\varepsilon_{k} / r_{h_{k}} \rightarrow 0^{+}$.

For any $k \in \mathbb{N}$ we choose a point $x_{k}$ in $\varepsilon_{k} \mathbb{Z}^{2}$ with minimal distance from $x$, and we define $\tilde{u}_{k}: Q \rightarrow \mathbb{R}$ by

$$
\tilde{u}_{k}(y):=u_{k}^{r_{h_{k}}}\left(y-\frac{x-x_{k}}{r_{h_{k}}}\right)=u_{k}\left(x_{k}+r_{h_{k}} y\right)
$$

The function $\tilde{u}_{k}$ is well defined: since $\left(x-x_{k}\right) / r_{h_{k}}$ tends to zero as $k \rightarrow+\infty$, for $k$ sufficiently large we have $y-\left(x-x_{k}\right) / r_{h_{k}} \in B_{1}(0)$ for every $y \in Q$. Note that $\tilde{u}_{k}$ tends to $\tilde{u}$ strongly in $L^{p}(Q)$ and $M S\left(\tilde{u}_{k}, Q\right)$ is bounded by (4.6). Moreover, as $S_{u_{k}} \subseteq \varepsilon_{k}\left(\left[\delta_{k}, 1-\delta_{k}\right]^{2}+\mathbb{Z}^{2}\right)$, we have $S_{\tilde{u}_{k}} \subseteq \tilde{\varepsilon}_{k}\left(\left[\delta_{k}, 1-\delta_{k}\right]^{2}+\mathbb{Z}^{2}\right)$. Since $\lim _{k} \delta_{k} / \tilde{\varepsilon}_{k}=$ $+\infty$, by (3.1) we should have $M S\left(\tilde{u}_{k}, Q\right) \rightarrow+\infty$, which contradicts the boundedness of $M S\left(\tilde{u}_{k}, Q\right)$. 


\section{The BRittle CASE}

In this section we prove the following result.

Theorem 5.1. Let $F_{\varepsilon}$ be defined as in (2.1). If $\vartheta=0$, then $F_{\varepsilon} \Gamma$-converges to $M S$ as $\varepsilon \rightarrow 0^{+}$.

We shall use the following approximation argument.

Lemma 5.2. (See [7, Theorem 3.9 and Corollary 3.11]). Let $R$ be an open rectangle and let $u \in S B V^{2}(R) \cap L^{\infty}(R)$. For every $\tau>0$, there exists a function $v \in S B V^{2}(R) \cap L^{\infty}(R)$ such that

(i) $v \in W^{1, \infty}\left(R \backslash \bar{S}_{v}\right)$ and $\mathcal{H}^{1}\left(\bar{S}_{v} \backslash S_{v}\right)=0$,

(ii) $\bar{S}_{v}$ is a finite union of disjoint closed segments in $R$,

(iii) $\|u-v\|_{L^{p}(R)}<\tau$,

(iv) $\|\nabla u-\nabla v\|_{L^{2}\left(R, \mathbb{R}^{2}\right)}<\tau$,

(v) $\left|\mathcal{H}^{1}\left(S_{u}\right)-\mathcal{H}^{1}\left(S_{v}\right)\right|<\tau$.

Let $\mathcal{W}(R)$ be the space of the functions $v \in S B V^{2}(R) \cap L^{\infty}(R)$, which satisfy (i) and (ii).

Proof of Theorem 5.1. The liminf inequality is a straight consequence of Remark 2.1, so we need only construct a recovery sequence $u_{k}$ for any $u \in \mathcal{U}^{p}(\Omega)$. Thanks to a truncation argument we can suppose that $u \in S B V^{2}(\Omega) \cap L^{\infty}(\Omega)$. Moreover, by a reflection argument with respect to the Lipschitz boundary of $\Omega$ (see [6, Theorems 3.1 and 8.1 , and the proof of Theorem 1.2]), we can extend $u$ to a suitable open bounded set $U \supseteq \bar{\Omega}$ in such a way that $u \in S B V^{2}(U) \cap L^{\infty}(U)$ and

$$
\mathcal{H}^{1}\left(S_{u} \cap \partial \Omega\right)=0 .
$$

Furthermore, given a rectangle $R$ containing $U$, we can once again extend $u$, this time to $R$, setting $u=0$ in $R \backslash U$. Of course we could assume that $U$ has Lipschitz boundary, so $u \in S B V^{2}(R) \cap L^{\infty}(R)$.

Thanks to the liminf inequality on $\Omega$ and $R \backslash \bar{\Omega}$, to construct a recovery sequence on $\Omega$ it is enough to find a sequence $u_{k}$ in $S B V^{2}(R)$ converging to $u$ in $L^{p}(R)$ such that

$$
\begin{aligned}
& S_{u_{k}} \subseteq \varepsilon_{k}\left(\left[\delta_{k}, 1-\delta_{k}\right]^{2}+\mathbb{Z}^{2}\right), \\
& \limsup _{k \rightarrow+\infty} M S\left(u_{k}, R\right) \leq M S(u, R) .
\end{aligned}
$$

Indeed, (5.1), (5.2), and (5.3) give

$$
\begin{aligned}
M S(u, \Omega) & +M S(u, R \backslash \bar{\Omega})=M S(u, R) \geq \limsup _{k \rightarrow+\infty} M S\left(u_{k}, R\right) \\
& \geq \limsup _{k \rightarrow+\infty} M S\left(u_{k}, \Omega\right)+\liminf _{k \rightarrow+\infty} M S\left(u_{k}, R \backslash \bar{\Omega}\right) \\
& \geq \limsup _{k \rightarrow+\infty} M S\left(u_{k}, \Omega\right)+M S(u, R \backslash \bar{\Omega}) .
\end{aligned}
$$

Since $M S(u, R \backslash \bar{\Omega})<+\infty$, this implies

$$
M S(u, \Omega) \geq \limsup _{k \rightarrow+\infty} M S\left(u_{k}, \Omega\right) .
$$

By Lemma 5.2, it is enough to obtain (5.2) and (5.3) when $u \in \mathcal{W}(R)$. According to the definition of $\mathcal{W}(R)$, there exist disjoint closed segments $S^{1}, \ldots, S^{m} \subseteq R$ such that 
$\bigcup_{i=1}^{m} S^{i}=\bar{S}_{u}$. If necessary, we may slightly modify $u$ in a neighborhood of each $S^{i}$ so that none of these segments are horizontal or vertical. This simplifies our exposition later, avoiding the possibility that $S^{i} \subseteq \mathbb{R} \times \varepsilon_{k} \mathbb{Z}$ or $S^{i} \subseteq \varepsilon_{k} \mathbb{Z} \times \mathbb{R}$, which would require an additional argument. We denote the length of $S^{i}$ by $l_{i}$ and the angle formed by $S^{i}$ with the $x_{1}$-axis by $\beta_{i} \in(0, \pi / 2) \cup(\pi / 2, \pi)$. Let $\varepsilon_{k} \rightarrow 0^{+}$and let $\delta_{k}:=\delta\left(\varepsilon_{k}\right)$. To construct a recovery sequence $u_{k}$, the idea is to smooth out the function $u$ in the unbreakable zone $R \backslash \varepsilon_{k}\left(\left[\delta_{k}, 1-\delta_{k}\right]^{2}+\mathbb{Z}^{2}\right)$.

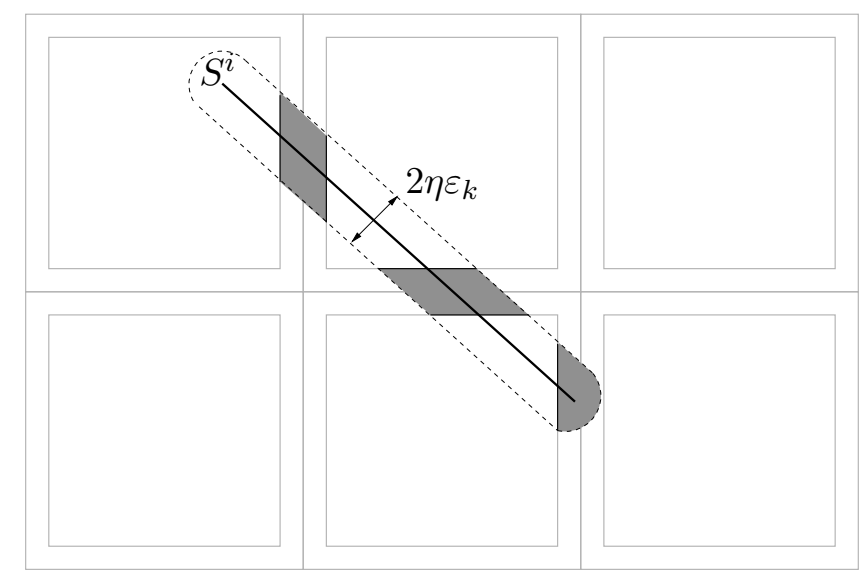

FiguRE 9

Fix a small $\eta>0$ and consider the sets $B_{\eta \varepsilon_{k}}\left(\bar{S}_{u}\right):=\left\{x \in \mathbb{R}^{2}: \operatorname{dist}\left(x, \bar{S}_{u}\right)<\eta \varepsilon_{k}\right\}$ (see Figure 9). There exists a constant $c<+\infty$, depending on $\bar{S}_{u}$, but not on $k$ and $\eta$, such that the restriction $\left.u\right|_{R \backslash B_{\eta \varepsilon_{k}}\left(\bar{S}_{u}\right)}$ has Lipschitz constant $c\left(\eta \varepsilon_{k}\right)^{-1}\|\nabla u\|_{L^{\infty}\left(R, \mathbb{R}^{2}\right)}$. Therefore we can find a Lipschitz extension $v_{k}$ to $R$ satisfying

$$
\begin{aligned}
\left\|v_{k}\right\|_{L^{\infty}(R)} & \leq\|u\|_{L^{\infty}(R)}, \\
\left\|\nabla v_{k}\right\|_{L^{\infty}\left(R, \mathbb{R}^{2}\right)} & \leq \frac{c}{\eta \varepsilon_{k}}\|\nabla u\|_{L^{\infty}\left(R, \mathbb{R}^{2}\right)} .
\end{aligned}
$$

Now we can obtain a recovery sequence $u_{k}$ for $u$ on $R$ by modifying the function $u$ on $B_{\eta \varepsilon_{k}}\left(\bar{S}_{u}\right) \backslash \varepsilon_{k}\left(\left[\delta_{k}, 1-\delta_{k}\right]^{2}+\mathbb{Z}^{2}\right)$ (the gray zones in Figure 9) in the following way:

$$
u_{k}(x):= \begin{cases}v_{k}(x) & \text { if } x \in B_{\eta \varepsilon_{k}}\left(\bar{S}_{u}\right) \backslash \varepsilon_{k}\left(\left[\delta_{k}, 1-\delta_{k}\right]^{2}+\mathbb{Z}^{2}\right), \\ u(x) & \text { elsewhere in } R .\end{cases}
$$

By construction $u_{k}$ belongs to $S B V^{2}(R)$ and $S_{u_{k}} \subseteq \varepsilon_{k}\left(\left[\delta_{k}, 1-\delta_{k}\right]^{2}+\mathbb{Z}^{2}\right)$. Note that $u_{k}$ could present additional cracks along $B_{\eta \varepsilon_{k}}\left(\bar{S}_{u}\right) \cap \varepsilon_{k}\left(\partial\left[\delta_{k}, 1-\delta_{k}\right]^{2}+\mathbb{Z}^{2}\right)$ (the vertical and horizontal boundary of the gray zones in Figure 9$)$.

Each segment $S^{i}$ intersects $\mathbb{R} \times \varepsilon_{k} \mathbb{Z}$ at most $l_{i}\left(\sin \beta_{i}\right) / \varepsilon_{k}+1$ times and intersects $\varepsilon_{k} \mathbb{Z} \times \mathbb{R}$ at most $l_{i}\left|\cos \beta_{i}\right| / \varepsilon_{k}+1$ times. For $\varepsilon_{k}$ small enough, this implies

$$
\begin{aligned}
& \mathcal{L}^{2}\left(B_{\eta \varepsilon_{k}}\left(\bar{S}_{u}\right) \backslash \varepsilon_{k}\left(\left[\delta_{k}, 1-\delta_{k}\right]^{2}+\mathbb{Z}^{2}\right)\right) \leq 9 \eta \delta_{k} \varepsilon_{k} \mathcal{H}^{1}\left(S_{u}\right), \\
& \mathcal{H}^{1}\left(B_{\eta \varepsilon_{k}}\left(\bar{S}_{u}\right) \cap \varepsilon_{k}\left(\partial\left[\delta_{k}, 1-\delta_{k}\right]^{2}+\mathbb{Z}^{2}\right)\right) \leq 9 \eta \mathcal{H}^{1}\left(S_{u}\right) .
\end{aligned}
$$


By (5.6), $\mathcal{L}^{2}\left(\left\{u \neq u_{k}\right\}\right) \rightarrow 0$, and, hence, by (5.4), $u_{k}$ tends to $u$ strongly in $L^{p}(R)$. Moreover, by (5.5) and (5.6) we get the estimate

$$
\int_{R}\left|\nabla u_{k}(x)\right|^{2} d x \leq \int_{R}|\nabla u(x)|^{2} d x+\frac{9 c^{2} \delta_{k}}{\eta \varepsilon_{k}} \mathcal{H}^{1}\left(S_{u}\right)\|\nabla u\|_{L^{\infty}\left(R, \mathbb{R}^{2}\right)}^{2},
$$

while by (5.7)

$$
\mathcal{H}^{1}\left(S_{u_{k}}\right) \leq \mathcal{H}^{1}\left(S_{u}\right)+9 \eta \mathcal{H}^{1}\left(S_{u}\right) .
$$

Finally, combining (5.8) and (5.9), and using the fact that $\delta_{k} / \varepsilon_{k} \rightarrow 0$, we obtain

$$
\limsup _{k \rightarrow+\infty} M S\left(u_{k}, R\right) \leq M S(u, R)+9 \eta \mathcal{H}^{1}\left(S_{u}\right)
$$

Being $\eta>0$ arbitrary, by a diagonal argument we obtain (5.3).

\section{ACKNOWLEDGMENTS}

This work is part of the Project "Variational problems with multiple scales" 2006, supported by the Italian Ministry of Education, University, and Research.

M. Barchiesi wishes to thank Filippo Cagnetti, Massimiliano Morini and Lucia Scardia for interesting discussions on the subject.

The research of M. Barchiesi was also supported by the Center for Nonlinear Analysis (NSF Grants No. DMS-0405343 and DMS-0635983). He is also grateful to the International School for Advanced Studies of Trieste for kind hospitality and support during periods where this work was undertaken.

\section{REFERENCES}

[1] L. Ambrosio, N. Fusco \& D. Pallara. Functions of bounded variation and free discontinuity problems, in the Oxford Mathematical Monographs. Oxford University Press, 2000.

[2] H. Attouch. Variational convergence for functions and operators, in the Applicable Mathematics Series. Pitman, Boston, 1984.

[3] G. Bouchitté, I. Fonseca \& L. Mascarenhas. A global method for relaxation. Arch. Rational Mech. Anal., 145 (1998), 51-98.

[4] A. Braides. A handbook of $\Gamma$-convergence. In Handbook of Differential Equations: Stationary Partial Differential Equations, volume 3, pages 101-213. Elsevier, Amsterdam, 2006.

[5] A. Braides, A. Defranceschi \& E. Vitali. Homogenization of free discontinuity problems. Arch. Rational Mech. Anal., 135 (1996), 297-356.

[6] F. Cagnetti \& L. Scardia. An extension theorem in $S B V$ and an application to the homogenization of the Mumford-Shah functional in perforated domains. Preprint SISSA, Trieste, 2008.

[7] G. Cortesani. Strong approximation of GSBV functions by piecewise smooth functions. Ann. Univ. Ferrara Sez. VII (N.S.), 43 (1998), 27-49.

[8] G. Dal Maso \& C.I. Zeppieri. Homogenization of fiber reinforced brittle materials: the intermediate case. Preprint SISSA, Trieste, 2009.

[9] E. De Giorgi \& L. Ambrosio. New functionals in the calculus of variations. Atti Accad. Naz. Lincei Rend. Cl. Sci. Fis. Mat. Natur., 82 (1988), 199-210.

[10] E. De Giorgi, M. Carriero \& A. Leaci. Existence theorem for a minimum problem with free discontinuity set. Arch. Rational Mech. Anal., 108 (1989), 195-218.

[11] M. Focardi, M.S. Gelli \& M. Ponsiglione. Fracture mechanics in perforated domains: a variational model for brittle porous media. Math. Models Methods Appl. Sci., to appear.

[12] L. Scardia. Damage as $\Gamma$-limit of microfractures in anti-plane linearized elasticity. Math. Models Methods Appl. Sci., 18 (2008), 1703-1740.

[13] L. Scardia. Damage as $\Gamma$-limit of microfractures in linearized elasticity under non-interpenetration constraint. Preprint SISSA, Trieste, 2007. 
(Marco Barchiesi) BCAM, Bizkaia Technology Park, Building 500, 48160 Derio, Spain E-mail address: barchiesi@bcamath.org

(Gianni Dal Maso) SISSA, Via Beirut 2-4, 34014, Trieste, Italy

E-mail address: dalmaso@sissa.it 